POS PROCEEDINGS

\title{
Recent investigations of direct $C P$ violation in $B$-meson decays at Belle
}

\section{Chia-Ling Hsu*t}

The University of Sydney

E-mail: chia-ling.hsuasydney.edu.au

We present the summary of recent studies of $B^{0} \rightarrow \eta \eta, B^{0} \rightarrow \pi^{0} \pi^{0}, B^{+} \rightarrow K^{+} K^{-} \pi^{+}$, and $B^{0} \rightarrow$ $K_{S}^{0} K^{+} \pi^{-}$charmless $B$ decays using a data sample of 772 million $B \bar{B}$ pairs collected at the Belle detector running at the $\Upsilon(4 S)$ resonance at the KEKB asymmetric-energy $e^{+} e^{-}$collider.

The 39th International Conference on High Energy Physics (ICHEP2018)

4-11 July 2018

Seoul, Korea

${ }^{*}$ Speaker.

${ }^{\dagger}$ On Behalf of the Belle Collaboration 


\section{Evidence of the decay $B^{0} \rightarrow \eta \eta$}

The $B^{0} \rightarrow \eta \eta$ decay is dominated by the $b \rightarrow u$ Cabibbo- and color-suppressed tree diagram and the $b \rightarrow d$ penguin diagram. The branching fraction of this decay is expected to be $(0.3-$ $3.1) \times 10^{-6}$ [2].This decay plays an important role in improving the flavor $\mathrm{SU}(3)$ calculations of $\left|S_{c c s}-S_{f}\right|$, where the final state $f$ is $\eta^{\prime} K$ or $\phi K$, the $C P$-violating parameter $S_{f} \sim \sin 2 \phi_{1}$ is measured in the time-dependent analysis [3], and the $C P$-violating parameter $S_{c \bar{c} s}$ is measured in the Cabibbo-Kobayashi-Maskawa(CKM)-favored $b \rightarrow c \bar{c} s$ decays. The bound on $\sin 2 \phi_{1}$ can be improved by the precise measurement of the branching fraction of $B^{0} \rightarrow \eta \eta$ [4]. This mode has been studied previously by Belle and BABAR [5, 6]. The best upper limit on this branching fraction is set to be $\mathscr{B}\left(B^{0} \rightarrow \eta \eta\right)<1.0 \times 10^{-6}$ at $90 \%$ confidence level (CL) by BaBar [6].

The $\eta_{3 \pi}$ candidates are reconstructed by two oppositely charged pions and a $\pi^{0}$ candidate. The $B^{0}$ candidates are identified using two kinematic variables in the center-of-mass frame: the beam constrained mass $M_{\mathrm{bc}} \equiv \sqrt{E_{\text {beam }}^{2}-p_{B}^{2}}$ and the energy difference $\Delta E \equiv E_{B}-E_{\text {beam }}$, where $E_{\text {beam }}$ is the beam energy, and $E_{B}$ and $p_{B}$ are the reconstructed energy and momentum of $B^{0}$ candidates. The dominant background is from the continuum process $e^{+} e^{-} \rightarrow q \bar{q}(q=u, d, s, c)$, which is suppressed with a neural network. We transform the neural network output $C_{\mathrm{NN}}$ to $C_{\mathrm{NN}}^{\prime}=\ln \left(\frac{C_{\mathrm{NN}}-C_{\mathrm{NN}}^{\min }}{C_{\mathrm{NN}}^{\max }-C_{\mathrm{NN}}}\right)$, where $C_{\mathrm{NN}}^{\min }$ is -0.8 and $C_{\mathrm{NN}}^{\max }$ is the maximum variables of the neural network outputs.

The branching fraction of $B^{0} \rightarrow \eta \eta$ is obtained by a simultaneous fit to the $\eta_{\gamma \gamma} \eta_{\gamma \gamma}, \eta_{\gamma \gamma} \eta_{3 \pi}$ and $\eta_{3 \pi} \eta_{3 \pi}$ decay channels. We perform a three dimensional extended unbinned maximum likelihood fit to the variables $M_{\mathrm{bc}}, \Delta E$ and $C_{\mathrm{NN}}^{\prime}$.

The measured branching fraction [7] is $\mathscr{B}\left(B^{0} \rightarrow \eta \eta\right)=\left(7.6_{-2.3-1.6}^{+2.7+1.4}\right) \times 10^{-7}$, where the first uncertainty is statistical and the second is systematic. The significance of the result is $3.3 \sigma$ above zero, and provides the first evidence of this decay.

\section{Measurements of branching fraction and $C P$ asymmetry on $B^{0} \rightarrow \pi^{0} \pi^{0}$}

The CKM angle $\phi_{2}$ can be determined by measuring the time-dependent $C P$ violation in $B \rightarrow$ $\pi \pi$ decays. Possible penguin contributions can give rise to direct $C P$ violation and potentially modify the asymmetry by introducing $\Delta \phi_{2}$ in the mixing-induced $C P$ violation parameter, $S_{C P}=$ $\sqrt{1-\mathscr{A}_{C P}^{2}} \sin \left[2\left(\phi_{2}-\Delta \phi_{2}\right)\right]$. An isospin analysis of the $B \rightarrow \pi \pi$ systems is needed to extract $\Delta \phi_{2}$ information [8].

The $B^{0} \rightarrow \pi^{0} \pi^{0}$ candidates from the subsequent decay of $\pi^{0}$ mesons to two photons. The dominant background arises from the continuum process. To suppress this, a Fisher discriminant $\left(T_{c}\right)$ from event shape variables. The signal yield and $\mathscr{A}_{C P}$ are extracted using an unbinned extended maximum likelihood fit to $M_{\mathrm{bc}}, \Delta E$, and $T_{c}$. We obtain a signal yield of $217 \pm 32$ events in the dataset. The branching fraction and $\mathscr{A}_{C P}$ determined to be $\mathscr{B}\left(B^{0} \rightarrow \pi^{0} \pi^{0}\right)=(1.31 \pm 0.19 \pm 0.19) \times$ $10^{-6}$ and $A_{C P}=+0.14 \pm 0.36 \pm 0.10$, where the quoted uncertainties are statistical and systematic, respectively. The measured branching fraction is consistent with the BABAR measurement [9] and supersedes the previous Belle one [10]. 
These results are combined with Belle's earlier measurements of $B \rightarrow \pi \pi[11,12,13]$ to exclude the $C P$-violating parameter $\phi_{2}$ from the range $15.5^{\circ}<\phi_{2}<75.0^{\circ}$ at $95 \%$ confidence level [13].

\section{Measurements of branching fraction and $C P$ asymmetry on $B^{+} \rightarrow K^{+} K^{-} \pi^{+}$}

Three-body charmless hadronic $B$ decays are suppressed in the standard model (SM) and are also sensitive to localized $C P$ violation in the phase space [14]. In recent years, unidentified structure has been measured by BaBar [15] and LHCb [16] in the $K^{+} K^{-}$low-invariant-mass spectrum of the $B^{+} \rightarrow K^{+} K^{-} \pi^{+}$decay. The LHCb studies revealed a non-zero inclusive $C P$ asymmetry of $-0.123 \pm 0.017 \pm 0.012 \pm 0.007$ and a large unquantified local $C P$ asymmetry in the same mass region. These results suggest that final-state interactions may be a contributing factor to $C P$ violation $[17,18]$.

The dominant background is from continuum $e^{+} e^{-} \rightarrow q \bar{q}(q=u, d, s, c)$ processes. A neural network is employed by combining variables based on the event topology in order to suppress the continuum background. Veto criteria are introduced to suppress the background contributions from $B$ decays via the dominant $b \rightarrow c$ transition. There are a few modes of $B$ decays via $b \rightarrow q(q=$ $u, d, s)$ processes that contribute in the $M_{\mathrm{bc}}$ signal region with a corresponding $\Delta E$ peak background. These peaking backgrounds are due to $K-\pi$ misidentification, including $B^{+} \rightarrow K^{+} K^{-} K^{+}, B^{+} \rightarrow$ $K^{+} \pi^{-} \pi^{+}$, and their intermediate resonant modes.

To investigate the localized $C P$ asymmetry in the low $M_{K^{+} K^{-}}$region, the signal yield and $\mathscr{A}_{C P}$ are extracted by performing a two-dimensional extended unbinned maximum likelihood fit in $M_{\mathrm{bc}}$ and $\Delta E$ in the bins of $M_{K^{+} K^{-}}$. The inclusive branching fraction is obtained by integrating the differential branching fraction over the entire mass range. The resulting branching fraction and direct $C P$ asymmetry are [19] $\mathscr{B}\left(B^{+} \rightarrow K^{+} K^{-} \pi^{+}\right)=(5.38 \pm 0.40 \pm 0.35) \times 10^{-6}$ and $\mathscr{A}_{C P}=$ $-0.170 \pm 0.073 \pm 0.017$, where the quoted uncertainties are statistical and systematic, respectively.

An excess and a large $\mathscr{A}_{C P}$ are seen in $M_{K^{+} K^{-}}<1.5 \mathrm{GeV} / c^{2}$, confirming the observations by $\mathrm{BaBar}$ and $\mathrm{LHCb}$. A strong evidence of large $C P$ asymmetry of $-0.90 \pm 0.17 \pm 0.03$ with $4.8 \sigma$ significance is found in the region of $M_{K^{+} K^{-}}<1.1 \mathrm{GeV} / c^{2}$.

\section{Measurements of branching fraction and $C P$ asymmetry on $B^{0} \rightarrow K_{S}^{0} K^{+} \pi^{-}$}

The decays with even number of kaons proceed via the $b \rightarrow u$ tree-level, the $b \rightarrow u W$ exchange, and the $b \rightarrow d$ penguin process with a virtual loop, which provides an opportunity to search for physics beyond the SM since new heavy particles may cause deviations from SM predictions. Previous measurements by the BaBar [20] and LHCb [21] experiments find hints of structures at the low $K^{-} \pi^{+}$and $K^{-} K_{S}^{0}$ regions that have highly asymmetric helicity angular distributions. However, the yield is not enough to draw firm conclusions with a full Dalitz analysis. Similar studies on $B^{+} \rightarrow K^{+} K^{-} \pi^{+}$were performed by Belle [19], BaBar [15], and LHCb [16], in which strong evidence of localized $C P$ violation was found in the low $M_{K^{+} K^{-}}$region.

Veto criteria are introduced to suppress the background contributions from $B$ decays via the dominant $b \rightarrow c$ transition. There are a few modes that contribute in the $M_{\mathrm{bc}}$ signal region with a 
corresponding $\Delta E$ peak background. These peaking backgrounds are due to $K-\pi$ misidentification, including $B^{0} \rightarrow K^{-} K^{+} K_{S}^{0}, B^{0} \rightarrow \pi^{-} \pi^{+} K_{S}^{0}$, and their intermediate resonant modes.

The signal yield and $\mathscr{A}_{C P}$ are extracted using an unbinned extended maximum likelihood fit to $M_{\mathrm{bc}}, \Delta E$, and $C_{\mathrm{NN}}^{\prime}$. The resulting branching fraction and direct $C P$ asymmetry are [22] $\mathscr{B}\left(B^{+} \rightarrow K^{+} K^{-} \pi^{+}\right)=(3.60 \pm 0.33 \pm 0.15) \times 10^{-6}$ and $\mathscr{A}_{C P}=(-8.5 \pm 8.9 \pm 0.2) \%$, where the quoted uncertainties are statistical and systematic, respectively.

Hints of peaking structures are seen around $1.2 \mathrm{GeV} / c^{2}$ of $M_{K^{-} K_{S}^{0}}$ and around $4.2 \mathrm{GeV} / c^{2}$ of $M_{\pi^{+} K_{S}^{0}}$ when compared to the phase space MC. No obvious $K^{*}$ structure is seen at both low $M_{K^{-} \pi^{+}}$ and $M_{\pi^{+} K_{S}^{0}}$ spectrums.

\section{References}

[1] K. A. Olive et al. (Particle Data Group), Chin. Phys. C 38, 090001 (2014).

[2] H.-Y. Chen and C.-K. Chua, Phys. Rev. D 80, 114008 (2009).

[3] B. Aubert et al. (BABAR Collaboration), Phys. Rev. D 79, 052003 (2009); K.-F. Chen et al. (Belle Collaboration), Phys. Rev. Lett. 98, 031802 (2007).

[4] M. Gronau, J. L. Rosner, and J. Zupan, Phys. Lett. B 596, 107 (2004).

[5] P. Chang et al. (Belle Collaboration), Phys. Rev. D 71, 091106 (2005).

[6] B. Aubert et al. (BABAR Collaboration), Phys. Rev. D 80, 112002 (2009).

[7] A. Abdesselam et al. (Belle Collaboration), arXiv:1609.03267 [hep-ex].

[8] M. Gronau and D. London, Phys. Rev. Lett. 65, 33813384 (1990).

[9] J. Lees et al. (BaBar Collaboration), Phys. Rev. D 87, 052009 (2013).

[10] Y. Chao et al. (Belle Collaboration), Phys. Rev. Lett. 94, 181803 (2005).

[11] Y.-T. Duh et al. (Belle Collaboration), Phys. Rev. D 87, 031103(R) (2012).

[12] J. Dalseno et al. (Belle Collaboration), Phys. Rev. D 88, 092003 (2013).

[13] T. Julius et al. (Belle Collaboration), Phys. Rev. D 96, 032007 (2017).

[14] I. Bediaga et al., Phys. Rev. D 86, 036005 (2012).

[15] B. Aubert et al. (BaBar Collaboration) Phys. Rev. Lett. 99, 221801 (2007).

[16] R. Aaij et al. (LHCb Collaboration) Phys. Rev. D 90, 112004 (2014).

[17] B. Bhattacharya, M. Gronau, and J. L. Rosner, Phys. Lett. B 726, 337 (2013).

[18] I. Bediaga, O. Lourenço, and T. Frederico, Phys. Rev. D 89, 094013 (2014).

[19] C.-L. Hsu et al., (Belle Collaboration), Phys. Rev. D 96, 031101 (2017).

[20] P. del Amo Sanchez et al., (BABAR Collaboration) Phys. Rev. D 82, 031101 (2010).

[21] Aaij, R. et al., (LHCb Collaboration) J. High Energy Phys. 11 (2017) 027

[22] A. Abdesselam et al., (Belle Collaboration), arXiv:1807.06782 [hep-ex]. 